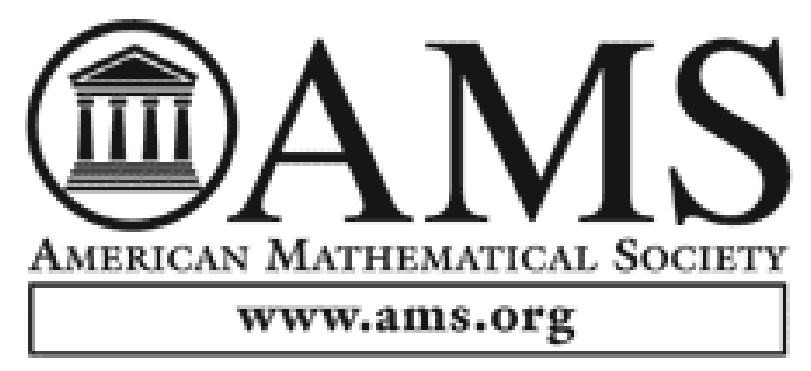

A Consistency Result on Thin-Tall Superatomic Boolean Algebras

Author(s): Juan Carlos Martínez

Source: Proceedings of the American Mathematical Society, Vol. 115, No. 2 (Jun., 1992), pp. 473-477

Published by: American Mathematical Society

Stable URL: http://www.jstor.org/stable/2159270

Accessed: 06/02/2009 06:55

Your use of the JSTOR archive indicates your acceptance of JSTOR's Terms and Conditions of Use, available at http://www.jstor.org/page/info/about/policies/terms.jsp. JSTOR's Terms and Conditions of Use provides, in part, that unless you have obtained prior permission, you may not download an entire issue of a journal or multiple copies of articles, and you may use content in the JSTOR archive only for your personal, non-commercial use.

Please contact the publisher regarding any further use of this work. Publisher contact information may be obtained at http://www.jstor.org/action/showPublisher?publisherCode=ams.

Each copy of any part of a JSTOR transmission must contain the same copyright notice that appears on the screen or printed page of such transmission.

JSTOR is a not-for-profit organization founded in 1995 to build trusted digital archives for scholarship. We work with the scholarly community to preserve their work and the materials they rely upon, and to build a common research platform that promotes the discovery and use of these resources. For more information about JSTOR, please contact support@jstor.org.

American Mathematical Society is collaborating with JSTOR to digitize, preserve and extend access to Proceedings of the American Mathematical Society. 


\title{
A CONSISTENCY RESULT ON THIN-TALL SUPERATOMIC BOOLEAN ALGEBRAS
}

\author{
JUAN CARLOS MARTÍNEZ
}

(Communicated by Andreas R. Blass)

\begin{abstract}
We prove that if $\varkappa$ is an infinite cardinal with $\varkappa^{<\varkappa}=\varkappa$, then there is a cardinal-preserving notion of forcing that forces the existence of a $\varkappa$-thin-tall superatomic Boolean algebra. Consistency for specific $\varkappa$, like $\omega_{1}$, then follows as a corollary.
\end{abstract}

A superatomic Boolean algebra (abbreviated $\mathrm{sBa}$ ) is a Boolean algebra in which every subalgebra is atomic. It is known that a Boolean algebra $B$ is superatomic iff its Stone space $S(B)$ is scattered. The Cantor-Bendixson process for topological spaces can be transferred to the context of Boolean algebras, obtaining in this way a sequence of ideals, which are called the Cantor-Bendixson ideals. Suppose that $B$ is a Boolean algebra. Then, for every ordinal $\alpha$, we define by transfinite induction, the ideal $I_{\alpha}$ as follows. We put $I_{0}=\{0\}$. If $\alpha=\beta+1$, let $I_{\alpha}$ be the ideal generated by $I_{\beta}$ together with all $b \in B$ such that $b / I_{\beta}$ is an atom in $B / I_{\beta}$. If $\alpha$ is limit, $I_{\alpha}=\bigcup\left\{I_{\beta}: \beta<\alpha\right\}$. Then, $B$ is an $\mathrm{sBa}$ iff $B=I_{\alpha}$ for some $\alpha$.

The height of an $\mathrm{sBa} B, \operatorname{ht}(B)$, is the least ordinal $\alpha$ such that $B / I_{\alpha}$ is finite (which means $\left.B=I_{\alpha+1}\right)$. For every $\alpha<\operatorname{ht}(B)$ let $\operatorname{wd}_{\alpha}(B)$ be the cardinality of the set of atoms in $B / I_{\alpha}$. The width of $B, \operatorname{wd}(B)$, is the supremum of the $\operatorname{wd}_{\alpha}(B)$ for $\alpha<\operatorname{ht}(B)$. Then, for every infinite cardinal $\varkappa, B$ is called $\varkappa$-thin-tall, if $\operatorname{ht}(B)=\varkappa^{+}$and $\operatorname{wd}(B)=\varkappa$.

The reader may find in [4] a wide list of results on superatomic Boolean algebras, as well as a discussion of equivalent definitions and basic facts. In particular, it is known that it is possible to construct an $\omega$-thin-tall sBa with no extra set-theoretic axioms. This was proved by Rajagopalan and, independently, by Juhász and Weiss. On the other hand, Baumgartner and Shelah proved in [1] that it is consistent with the axioms of set theory that there exists an $\mathrm{sBa} B$ such that $\operatorname{ht}(B)=\omega_{2}$ and $\operatorname{wd}(B)=\omega$. The argument employed by Baumgartner and Shelah uses the fact that the forcing conditions are finite. However, if we want to prove, for an uncountable cardinal $\varkappa$, that the existence of a $\varkappa$-thin-tall $\mathrm{sBa}$ is consistent with the axioms of set theory, then we have to consider infinite forcing conditions. In this paper we see a modification of the argument given in [1], which permits us to deal with infinite forcing conditions. The set-theoretic

Received by the editors February 12, 1990 and, in revised form, November 14, 1990.

1980 Mathematics Subject Classification (1985 Revision). Primary 03E35, 06 E99. 
terminology used here is taken from [1]. Our aim is to prove the following result.

Theorem. If $\varkappa$ is an infinite cardinal with $\varkappa^{<\varkappa}=\varkappa$, then there is a cardinalpreserving notion of forcing that forces the existence of a $\varkappa$-thin-tall $s B a$.

Proof. Let $\varkappa$ be an infinite cardinal with $\varkappa^{<\varkappa}=\varkappa$. Note that this implies, by König's lemma, that $\varkappa$ is regular. We define a partial ordering $P_{\varkappa}$, which does not depend on any special function, and we prove that forcing with $P_{\varkappa}$ preserves cardinals and adjoins a $\varkappa$-thin-tall sBa.

We put $T=\varkappa^{+} \times \varkappa$ and, for every $\alpha<\varkappa^{+}, T_{\alpha}=\{\alpha\} \times \varkappa . P_{\varkappa}$ adjoins a partial ordering $\leq$ on $T$ and a function $i$ on the set $\{\{s, t\}: s, t \in T\}$ such that the supremum of $i\{s, t\}$ represents the meet $s \wedge t$. We define $P_{\varkappa}$ as the set of all $p=\left(x_{p}, \leq_{p}, i_{p}\right)$ satisfying the following conditions:

(1) $x_{p}$ is a subset of $T$ of cardinality $<\varkappa$.

(2) $\leq_{p}$ is a partial ordering of $x_{p}$ such that if $s \in T_{\alpha}, t \in T_{\beta}$, and $s<_{p} t$, then $\alpha<\beta$.

(3) $i_{p}:\left\{\{s, t\}: s, t \in x_{p}\right\} \rightarrow\left\{x: x\right.$ is a finite subset of $\left.x_{p}\right\}$ satisfies the following:

(3.1) If $s \in T_{\alpha}, t \in T_{\beta}$, and $\alpha \leq \beta$ then:

(3.1.1) If $s=t$, then $i_{p}\{s, t\}=\{s\}$.

(3.1.2) If $s \neq t$ and $\alpha=\beta$, then $i_{p}\{s, t\}=0$.

(3.1.3) If $s<_{p} t$, then $i_{p}\{s, t\}=\{s\}$

(3.1.4) If $\alpha<\beta$ and $s \not_{p} t$, then $i_{p}\{s, t\} \subseteq x_{p} \cap \bigcup\left\{T_{\tau}: \tau<\alpha\right\}$.

(3.2) For every $s, t \in x_{p}$ the following hold:

(3.2.1) If $u \in i_{p}\{s, t\}$, then $u \leq_{p} s, t$.

(3.2.2) If $v \leq_{p} s, t$, then there is a $u \in i_{p}\{s, t\}$ with $v \leq_{p} u$.

Now we put $p \leq_{\varkappa} q$ iff $x_{p} \supseteq x_{q}, \leq_{p}\left\lceil x_{q}=\leq_{q}\right.$ and $\ddot{l}_{p} \uparrow\left\{\{s, t\}: s, t \in x_{q}\right\}=$ $i_{q}$.

Then proceeding in a way similar to that for [1, Theorem 7.1], one can prove that if $P_{\varkappa}$ preserves cardinals, then $P_{\varkappa}$ adjoints a $\varkappa$-thin-tall sBa.

Our aim is to show that forcing with $P_{\varkappa}$ preserves cardinals. Note that $P_{\varkappa}$ is $\varkappa$-closed. Then, our purpose is to prove that $P_{\varkappa}$ satisfies the $\varkappa^{+}$-chain condition. Suppose on the contrary that there exists an antichain $A$ of cardinality $\varkappa^{+}$. For every $p \in A$, we put $\gamma_{p}=\left\{\alpha: x_{p} \cap T_{\alpha} \neq 0\right\}$. Then, by the $\Delta$-system lemma (see [3, Theorem II.1.6]), we may assume that the $\gamma_{p}$ form a $\Delta$-system with kernel $\Delta$. Since the cardinality of every $\gamma_{p}$ is $<\varkappa$ and, for all $\alpha, \beta \in \Delta$ with $\alpha<\beta$, the cardinality of $\beta-\alpha$ is $\leq \varkappa$, we may also assume that $\Delta$ is an initial segment of $\gamma_{p}$ for every $p \in A$. Then by thinning out $A$ again if necessary, we may suppose that there is an ordinal $\gamma^{(1)}<\varkappa$ such that the order type of $\gamma_{p}-\Delta$ is $\gamma^{(1)}$ for every $p \in A$. Now we define $\gamma^{(0)}=$ supremum $\{\alpha+1: \alpha \in \Delta\}$, and $\gamma=\left(\gamma^{(0)}+\gamma^{(1)}\right)-\gamma^{(0)}$. Note that, since the cardinality of $\gamma$ is $\left\langle\varkappa\right.$, we may assume that $\gamma_{p} \cap \gamma=0$ for every $p \in A$. Now, for every $p, q \in A$, we consider the unique order-preserving bijection $\pi_{p q}: \gamma_{p} \rightarrow \gamma_{q}$. Then, since the cardinality of each $x_{p}$ is $<\varkappa$, we may suppose that $\pi_{p q}$ lifts to an isomorphism of $x_{p}$ with $x_{q}$ given by $\pi_{p q}(\alpha, \beta)=\left(\pi_{p q}(\alpha), \beta\right)$. Finally we may also assume that, for every $p, q \in A$ and $s, t \in x_{p}$, we have:

$$
s \leq_{p} t \quad \text { iff } \pi_{p q}(s) \leq_{q} \pi_{p q}(t)
$$


and

$$
i_{q}\left(\pi_{p q}(s), \pi_{p q}(t)\right)=\left\{\pi_{p q}(u): u \in i_{p}\{s, t\}\right\} .
$$

Now we prove that the elements of $A$ are all compatible. Let $p, q \in A$. We construct an $r \in P_{\varkappa}$ such that $r \leq_{x} p$ and $r \leq_{\varkappa} q$. Let $\rho: \gamma \rightarrow\left(\gamma_{p}-\Delta\right)$ and $\mu: \gamma \rightarrow\left(\gamma_{q}-\Delta\right)$ the corresponding order-preserving bijections. For each $\alpha \in \gamma$, we set

$$
x^{(\alpha)}=\left\{(\alpha, \beta) \in T_{\alpha}:(\rho(\alpha), \beta) \in x_{p}\right\}=\left\{(\alpha, \beta) \in T_{\alpha}:(\mu(\alpha), \beta) \in x_{q}\right\} .
$$

Then we put

$$
x_{r}=x_{p} \cup x_{q} \cup \bigcup\left\{x^{(\alpha)}: \alpha \in \gamma\right\} .
$$

Now we make the following definitions:

$$
\begin{aligned}
x & =\bigcup\left\{x_{p} \cap T_{\alpha}: \alpha \in \Delta\right\}=\bigcup\left\{x_{q} \cap T_{\alpha}: \alpha \in \Delta\right\}, \\
y & =\bigcup\left\{x^{(\alpha)}: \alpha \in \gamma\right\}, \\
z_{1} & =\bigcup\left\{x_{p} \cap T_{\alpha}: \alpha \in \gamma_{p}-\Delta\right\}, \\
z_{2} & =\bigcup\left\{x_{q} \cap T_{\alpha}: \alpha \in \gamma_{q}-\Delta\right\} .
\end{aligned}
$$

Note that $\rho$ and $\mu$ lift to the isomorphisms of $y$ with $z_{1}$ and $y$ with $z_{2}$, respectively, given by $\rho(\alpha, \beta)=(\rho(\alpha), \beta)$ and $\mu(\alpha, \beta)=(\mu(\alpha), \beta)$. Then we define $\leq_{r}$ as follows: $s \leq_{r} t$ iff $s \leq_{p} t$ or $s \leq_{q} t$ or one of the following conditions holds:

(a) $s \in x, t \in y$, and $s \leq_{p} \rho(t)$;

(b) $s, t \in y$ and $\rho(s) \leq_{p} \rho(t)$;

(c) $s \in y, t \in z_{1}$, and $\rho(s) \leq_{p} t$;

(d) $s \in y, t \in z_{2}$, and $\mu(s) \leq_{q} t$.

We show that $\leq_{r}$ is a transitive order. Suppose that $s \leq_{r} t \leq_{r} u$. The cases $s, t, u \in x_{p}$ and $s, t, u \in x_{q}$ are obvious. If $s \in x, t \in y$, and $u \in z_{1}$, we have that $s \leq_{p} \rho(t) \leq_{p} u$, whence $s \leq_{r} u$. Suppose that $s, t \in y$ and $u \in z_{1}$. It follows that $\rho(s) \leq_{p} \rho(t) \leq_{p} u$, and hence $s \leq_{r} u$. Now assume that $s, t \in y$ and $u \in z_{2}$. Since $\rho(s) \leq_{p} \rho(t)$, we have $\pi_{p q}(\rho(s)) \leq_{q} \pi_{p q}(\rho(t))$. But note that $\pi_{p q}(\rho(s))=\mu(s)$ and $\pi_{p q}(\rho(t))=\mu(t)$. Therefore $\mu(s) \leq_{q} \mu(t) \leq_{q} u$, and so $s \leq_{r} u$. The other cases are proved in a similar way.

Now we define $i_{r}$. Let $s, t \in x_{r}$. If $s, t \in x_{p}$, we put $i_{r}\{s, t\}=i_{p}\{s, t\}$. If $s, t \in x_{q}$, then $i_{r}\{s, t\}=i_{q}\{s, t\}$. If $s \in x$ and $t \in y$, we set $i_{r}\{s, t\}=$ $i_{p}\{s, \rho(t)\}$. If $s, t \in y$, then $i_{r}\{s, t\}=\left(i_{p}\{\rho(s), \rho(t)\} \cap x\right) \cup\left\{\rho^{-1}(u): u \in\right.$ $\left.i_{p}\{\rho(s), \rho(t)\}-x\right\}$. If $s \in y$ and $t \in z_{1}$, then $i_{r}\{s, t\}=i_{r}\left\{s, \rho^{-1}(t)\right\}$. Analogously if $s \in y$ and $t \in z_{2}$, then $i_{r}\{s, t\}=i_{r}\left\{s, \mu^{-1}(t)\right\}$. Finally, if $s \in z_{1}$ and $t \in z_{2}$, we put $i_{r}\{s, t\}=i_{r}\left\{\rho^{-1}(s), \mu^{-1}(t)\right\}$.

Note that $i_{r}$ is well defined. For example, if $s \in z_{1}, t \in z_{2}$, and $\rho^{-1}(s)=$ $\mu^{-1}(t)$, then $i_{r}\{s, t\}=\left\{\rho^{-1}(s)\right\}$. On the other hand, it should be noted that if $s \in y$ and $t \in z_{1}$, then $i_{r}\{s, t\}=\left(i_{p}\{\rho(s), t\} \cap x\right) \cup\left\{\rho^{-1}(u): u \in i_{p}\{\rho(s), t\}-\right.$ $x\}$, and analogously, if $s \in y$ and $t \in z_{2}$, then $i_{r}\{s, t\}=\left(i_{q}\{\mu(s), t\} \cap x\right) \cup$ $\left\{\mu^{-1}(u): u \in i_{q}\{\mu(s), t\}-x\right\}$.

In order to show that $r=\left(x_{r}, \leq_{r}, i_{r}\right) \in P_{\varkappa}$, we must verify condition (3). The easy proof of (3.1) is left to the reader. We prove condition (3.2.1). Let $s, t \in x_{r}$ and $u \in i_{r}\{s, t\}$. The cases $s, t \in x_{p}$ and $s, t \in x_{q}$ are obvious. For the rest, we consider three cases.

Case 1. $s \in x$ and $t \in y$. Then $u \in i_{p}\{s, \rho(t)\}$, and therefore $u \leq_{p} s, \rho(t)$, whence $u \leq_{r} s, t$. 
Case 2. $s, t \in y$. If $u \in x$, then $u \in i_{p}\{\rho(s), \rho(t)\}$, and hence $u \leq_{p}$ $\rho(s), \rho(t)$, whence $u \leq_{r} s, t$.

If $u \in y$, then $\rho(u) \in i_{p}\{\rho(s), \rho(t)\}$, and so $\rho(u) \leq_{p} \rho(s), \rho(t)$, which implies $u \leq_{r} s, t$.

If $s \in y$ and $t \in z_{1}$, or $s \in y$ and $t \in z_{2}$, the considerations are similar to those of Case 2.

Case 3. $s \in z_{1}$ and $t \in z_{2}$. We have that $u \in i_{r}\left\{\rho^{-1}(s), \mu^{-1}(t)\right\}$ and then by Case 2, $u \leq_{r} \rho^{-1}(s), \mu^{-1}(t)$. If $u \in x$, it is clear that $u \leq_{r} s, t$. If $u \in y$, we infer than $\rho(u) \leq_{p} s$ and $\mu(u) \leq_{q} t$, which implies $u \leq_{r} s, t$.

Now we check (3.2.2). Let us consider $s, t \in x_{r}$ and $v \leq_{r} s, t$. The case $s \in X$ and $t \in x_{p} \cup x_{q}$ is obvious. For the rest, we consider four cases.

Case 1. $s \in x$ and $t \in y$. It follows that $v \leq_{p} s, \rho(t)$, and thus there is a $u \in i_{p}\{s, \rho(t)\}=i_{r}\{s, t\}$ such that $v \leq_{r} u$.

Case 2. $s, t \in y$. First suppose that $v \in x$. Then $v \leq_{p} \rho(s), \rho(t)$, and therefore there is a $u \in i_{p}\{\rho(s), \rho(t)\}$ such that $v \leq_{p} u$. If $u \in x$, then $u \in i_{r}\{s, t\}$. And if $u \in z_{1}$, we infer that $v \leq_{r} \rho^{-1}(u)$ and $\rho^{-1}(u) \in i_{r}\{s, t\}$.

Now suppose that $v \in y$. Then $\rho(v) \leq_{p} \rho(s), \rho(t)$, and hence there is a $u \in i_{p}\{\rho(s), \rho(t)\}$ such that $\rho(v) \leq_{p} u$, whence $v \leq_{r} \rho^{-1}(u)$ and $\rho^{-1}(u) \in$ $i_{r}\{s, t\}$.

The cases $s \in y, t \in z_{1}$ and $s \in y, t \in z_{2}$ can be verified by means of an argument similar to the one given in Case 2 .

Case 3. $s, t \in z_{1}$. If $v \in x_{p}$ we are done. Then suppose that $v \in y$. It follows that $\rho(v) \leq_{p} s, t$, and hence there is a $u \in i_{p}\{s, t\}$ such that $\rho(v) \leq_{p}$ $u$. But $\rho(v) \leq_{p} u$ implies $v \leq_{r} u$.

The case $s, t \in z_{2}$ is similar to Case 3 .

Case 4. $s \in z_{1}$ and $t \in z_{2}$. It is easy to infer that $v \leq_{r} \rho^{-1}(s), \mu^{-1}(t)$, and then by Case 2, there is a $u \in i_{r}\left\{\rho^{-1}(s), \mu^{-1}(t)\right\}=i_{r}\{s, t\}$ such that $v \leq_{r} u$.

This completes the verification of (3) and the proof that $P_{\varkappa}$ has the $\varkappa^{+}$-chain condition.

Remarks. (1) Juhász and Weiss proved in [2] that, for every ordinal $\alpha<\omega_{2}$, there exists an $\mathrm{sBa} B_{\alpha}$ such that $\operatorname{ht}\left(B_{\alpha}\right)=\alpha$ and $\operatorname{wd}\left(B_{\alpha}\right)=\omega$. Then, by using the well-known fact that there is an almost disjoint family of $2^{\omega}$ subsets of $\omega$, we obtain that $1 \mathrm{CH}$ implies the existence of an sBa with exactly $\omega$ atoms and height $\omega_{2}$. On the other hand, since the partial ordering $P_{\omega_{1}}$ is countably closed, we infer that forcing with $P_{\omega_{1}}$ preserves $\mathrm{CH}$ (see [3, Theorem VII.6.14]), and thus we obtain as a corollary that the existence of an $\omega_{1}$-thin-tall sBa is consistent with $\mathrm{ZFC}+\mathrm{CH}$.

(2) Suppose that $\varkappa, \lambda$ are infinite cardinals such that $\varkappa^{<\varkappa}=\varkappa$ and $\varkappa<\lambda$. Then, it is consistent with the axioms of set theory that there exists an $\mathrm{sBa} B$ such that $\operatorname{ht}(B)=\varkappa+1, \operatorname{wd}_{\alpha}(B)=\varkappa$ for every $\alpha<\varkappa$ and $\operatorname{wd}_{\varkappa}(B)=\lambda$. This result can be proved by means of an argument similar to the one given before. However, if we assume that the ground model satisfies $\mathrm{GCH}$, it is easier to show this fact, if we use the argument given in [5, Theorem 9]. More precisely, let us consider a cardinal-preserving generic extension $N$ such that, in $N, 2^{\varkappa} \geq \lambda$ and $2^{\varkappa_{0}}=\varkappa_{0}^{+}$for every cardinal $\varkappa_{0}<\varkappa$ (see [3, Theorem VII.6.17]). Then in $N$, the complete binary tree of height $\varkappa$ is a $\varkappa$-Canadian tree with at least $\lambda$ paths. But note that the existence of such a tree implies the existence of the required $\mathrm{sBa}$. 


\section{ACKNOWLEDGMENT}

I wish to express my gratitude to I. Juhász for bringing the problem considered here to my attention.

\section{REFERENCES}

1. J. Baumgartner and S. Shelah, Remarks on superatomic Boolean algebras, Ann. Pure Appl. Logic 33 (1987), 109-129.

2. I. Juhász and W. Weiss, On thin-tall scattered spaces, Colloq. Math. 90 (1978), 64-68.

3. K. Kunen, Set theory: an introduction to independence proofs, North-Holland, Amsterdam, 1980.

4. J. Roitman, Superatomic Boolean algebras, Handbook of Boolean Algebras (J. D. Monk and R. Bonnet, eds.), North-Holland, Amsterdam, 1989, pp. 719-740.

5. M. Weese, On cardinal sequences of Boolean algebras, Algebra Universalis 23 (1986), 85-97.

Facultad de Matemáticas, Universidad de Barcelona, 08007 Barcelona, Spain 